\title{
INTRODUCTION \\ Translational research advances in the evaluation and management of moyamoya disease
}

\author{
Edward R. Smith, MD, ${ }^{1}$ Giuseppe Lanzino, MD, ${ }^{2}$ Gary K. Steinberg, MD, $\mathrm{PhD},{ }^{3}$ and \\ $\mathrm{Bin} \mathrm{Xu}, \mathrm{MD}, \mathrm{PhD}^{4}$ \\ 1Department of Neurosurgery, Boston Children's Hospital, Boston, Massachusetts; '2Department of Neurosurgery, Mayo \\ Clinic, Rochester, Minnesota; ${ }^{3}$ Department of Neurosurgery, Stanford University School of Medicine, Stanford, California; and \\ ${ }^{4}$ Department of Neurosurgery, Huashan Hospital, Shanghai, China
}

$\mathrm{M}$ OYAMOYA disease has increasingly been recognized as a heterogeneous group of arteriopathies that share common clinical and radiographic features. Despite these similarities, significant variability in disease progression and response to treatment persist, highlighting the need for accelerated research efforts to improve the diagnostic, prognostic, and therapeutic outcomes for moyamoya patients.

This issue of Neurosurgical Focus includes a wide range of articles from international contributors showcasing advances in our understanding of moyamoya disease. These studies include work on genetic mutations, such as original transcriptome analysis implicating a potential role for the immune system in the pathogenesis of the disease. Important for any translational research is the development and utilization of animal models - crucial for both mechanistic and therapeutic projects. A current pair of complementary state-of-the-art reports provides the reader with valuable resources on this subject. Novel research efforts into medical and other nonsurgical treatments for moyamoya provide potential future directions for the field.
This issue of Neurosurgical Focus is important not only for the insight it provides specifically into moyamoya but also because it provides tangible examples of the impact that neurosurgeon-scientists have on translational research in general. Neurosurgeons remain uniquely qualified to serve as leaders in studying the conditions they treat. The moyamoya disease-related research reported here reinforces the critical value of neurosurgical translational research; much has been accomplished, but much more remains to be done.

https://thejns.org/doi/abs/10.3171/2021.6.FOCUS21373

\section{Disclosures}

The authors report no conflict of interest.

\section{Correspondence}

Edward R. Smith: edward.smith@childrens.harvard.edu. 